\title{
A two-step actin polymerization mechanism drives dendrite branching
}

\author{
Rebecca Shi ${ }^{1,2}$, Daniel A. Kramer ${ }^{3}$, Baoyu Chen ${ }^{3}$ and Kang Shen ${ }^{1,4^{*}}$ (i)
}

\begin{abstract}
Background: Dendrite morphogenesis plays an essential role in establishing the connectivity and receptive fields of neurons during the development of the nervous system. To generate the diverse morphologies of branched dendrites, neurons use external cues and cell surface receptors to coordinate intracellular cytoskeletal organization; however, the molecular mechanisms of how this signaling forms branched dendrites are not fully understood.

Methods: We performed in vivo time-lapse imaging of the PVD neuron in C. elegans in several mutants of actin regulatory proteins, such as the WAVE Regulatory Complex (WRC) and UNC-34 (homolog of Enabled/Vasodilator-stimulated phosphoprotein (EnaNASP)). We examined the direct interaction between the WRC and UNC-34 and analyzed the localization of UNC-34 in vivo using transgenic worms expressing UNC-34 fused to GFP.
\end{abstract}

Results: We identify a stereotyped sequence of morphological events during dendrite outgrowth in the PVD neuron in C. elegans. Specifically, local increases in width ("swellings") give rise to filopodia to facilitate a "rapid growth and pause" mode of growth. In unc-34 mutants, filopodia fail to form but swellings are intact. In WRC mutants, dendrite growth is largely absent, resulting from a lack of both swelling and filopodia formation. We also found that UNC-34 can directly bind to the WRC. Disrupting this binding by deleting the UNC-34 EVH1 domain prevented UNC-34 from localizing to swellings and dendrite tips, resulting in a stunted dendritic arbor and reduced filopodia outgrowth.

Conclusions: We propose that regulators of branched and linear F-actin cooperate to establish dendritic branches. By combining our work with existing literature, we propose that the dendrite guidance receptor DMA-1 recruits the WRC, which polymerizes branched F-actin to generate "swellings" on a mother dendrite. Then, WRC recruits the actin elongation factor UNC-34/EnaNASP to initiate growth of a new dendritic branch from the swelling, with the help of the actin-binding protein UNC-115/abLIM. Extension of existing dendrites also proceeds via swelling formation at the dendrite tip followed by UNC-34-mediated outgrowth. Following dendrite initiation and extension, the stabilization of branches by guidance receptors further recruits WRC, resulting in an iterative process to build a complex dendritic arbor.

Keywords: Dendrite branching, Dendrite morphogenesis, Actin polymerization, Ena/NASP, WAVE regulatory complex

\section{Background}

Neurite outgrowth and arborization are essential for the establishment and function of neural circuits. In particular, the morphology of dendrites is key for neuronal signal transmission and integration. Dendrite morphogenesis is

\footnotetext{
*Correspondence: kangshen@stanford.edu

${ }^{1}$ Department of Biology, Stanford University, Stanford, CA 94305, USA

Full list of author information is available at the end of the article
}

often guided by extracellular cues recognized through a number of cell surface receptors, such as Semaphorins, DSCAMs, and protocadherins, which in turn instruct activities of various intracellular molecules leading to cytoskeletal reorganization [1, 2]. Similarly, activitydependent dendritic arbor development also requires cytoskeletal signaling, including through Rho GTPase activity [3] or microtubule stabilization [4].

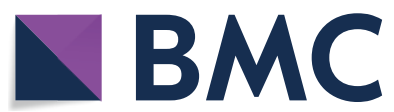

(c) The Author(s) 2021. Open Access This article is licensed under a Creative Commons Attribution 4.0 International License, which permits use, sharing, adaptation, distribution and reproduction in any medium or format, as long as you give appropriate credit to the original author(s) and the source, provide a link to the Creative Commons licence, and indicate if changes were made. The images or other third party material in this article are included in the article's Creative Commons licence, unless indicated otherwise in a credit line to the material. If material is not included in the article's Creative Commons licence and your intended use is not permitted by statutory regulation or exceeds the permitted use, you will need to obtain permission directly from the copyright holder. To view a copy of this licence, visit http://creativecommons.org/licenses/by/4.0/. The Creative Commons Public Domain Dedication waiver (http://creativeco mmons.org/publicdomain/zero/1.0/) applies to the data made available in this article, unless otherwise stated in a credit line to the data. 
One major signaling axis for neurite outgrowth is the activation and membrane recruitment of the actin nucleation promotion factor, the WAVE Regulatory Complex (WRC), a pentameric complex comprising Sra1/Cyfip1, Nap1/Hem-2, Abi, HSPC300, and WAVE/Scar [5]. After being activated and recruited by diverse membrane receptors and the Rho family GTPase Rac1, the WRC can then stimulate the Arp2/3 complex to produce branched actin networks [6]. In addition, a distinct actin elongation factor, Ena/VASP, can be recruited downstream of the Netrin receptor, DCC, [7-9] to promote polarized filopodia formation during axon guidance. Ena/VASP can also be recruited by the Slit receptor, Robo, to mediate axon repulsion $[10,11]$. Interestingly, these distinct actin regulators can act synergistically in several morphological processes. For example, the direct interaction between the WRC and Ena/VASP was shown to play an important role in photoreceptor axon targeting, oogenesis, and macrophage migration in Drosophila, lamellipodia formation during ventral closure of C. elegans embryogenesis, and collective axon extension in mice [12-14]. It is, however, largely unknown how the activity of distinct actin regulators is coordinated in complex morphological processes.

One hypothesis is that complex processes like neuronal morphogenesis integrate structurally distinct actin networks built by different actin regulators. For example, in dendritic spines of hippocampal pyramidal neurons, patches of loosely bundled linear actin filaments constitute the dendritic spine neck, whereas branched actin networks drive the expansion of spine heads [15]. Furthermore, Abl tyrosine kinase has been shown to downregulate Ena activity while in parallel activating Rac/ WAVE signaling, thus coordinating the balance of linear actin bundles and branched actin networks to regulate Drosophila axon patterning [16]. Finally, branched actin networks generated via the Arp $2 / 3$ complex were found to provide sites for initiating new dendrite branches in Drosophila larval sensory da neurons [17]. Therefore, it is likely neurons control morphological changes by dynamically orchestrating the formation of distinct types of actin networks in a coherent manner.

In this work, we provide evidence in support of this hypothesis showing that in the C. elegans PVD neuron, the development of dendritic arbors requires two distinct, but cooperative, steps of actin assembly that involve the WRC, UNC-34 (Ena/VASP), and the actin-binding protein UNC-115 (abLIM). As a recently developed model system for studying dendritic morphogenesis, the PVD neuron elaborates complex dendritic arbors by extending a primary dendrite along the length of the worm, followed by orthogonal secondary, tertiary, and quaternary dendrites, which together create stereotyped "menorah"-like structures [18]. A multipartite ligand-receptor complex, consisting of the extracellular ligands SAX-7, MNR-1, and LECT-2, along with the PVD guidance receptor DMA-1 and its partner HPO-30, directs the growth of PVD dendrites along the epidermis and body wall muscles [19-23]. This ligand-receptor complex recruits actin regulators, including the Rac GEF TIAM-1 and the WRC, through the cytosolic domains of DMA-1 and HPO-30, respectively, which in turn produce $\mathrm{F}$-actin in growing dendrite tips to drive dendrite branching [23-25]. However, the mechanisms of F-actin recruitment during distinct morphological steps during dendrite development, such as initiation of new branches and branch elongation, remain unclear.

Here, we show that new dendrite branch points are established first as local "swellings" along dendrites, from which filopodia sprout to enable rapid and efficient outgrowth of dendritic branches. The formation of swellings is mediated by the WRC, while the subsequent extension of filopodial branches is mediated by UNC-34/Ena/VASP and UNC-115/abLIM. Our data suggest that a direct interaction between the WRC and UNC-34 provides the mechanism by which two distinct actin assembling processes are coupled to drive dendrite arborization.

\section{Results \\ Morphological characterization of "swellings" and filopodia during PVD dendrite outgrowth}

To identify distinct morphological events during the development of PVD dendritic arbors, we performed in vivo live imaging of the PVD dendrite at the third larval stage (L3) of wild-type worms. At this stage, the dendrite elaborates its tertiary and quaternary branches (see Additional files 1 and 4). Using a myristoylated-GFP (myr-GFP) marker expressed specifically in PVD, we observed many dynamic growth and retraction events that ultimately led to the formation of menorah-like branches, consistent with previous findings $[18,26]$. In addition, we noticed that the growth of the quaternary branches often occurred through a series of rapid filopodia-like growth events, which were interspersed by several pauses (Fig. 1A-C). When we plotted the growth of quaternary dendrites over time (Fig. 1B, three independent examples) and examined dendrite morphology during growth events, we found that existing dendrites often exhibited a local increase in width (blue bars) right before the initiation of new branches or further extension of existing dendrites. Quantification of dendrite width showed a significant increase 1-2 min before new branch initiation (Fig. 1C), and filopodia emerged rapidly from these enlarged locations (Fig. 1B-C). We herein refer to these local increases in width as "swellings". Using a LifeAct:GFP marker, we confirmed that F-actin 


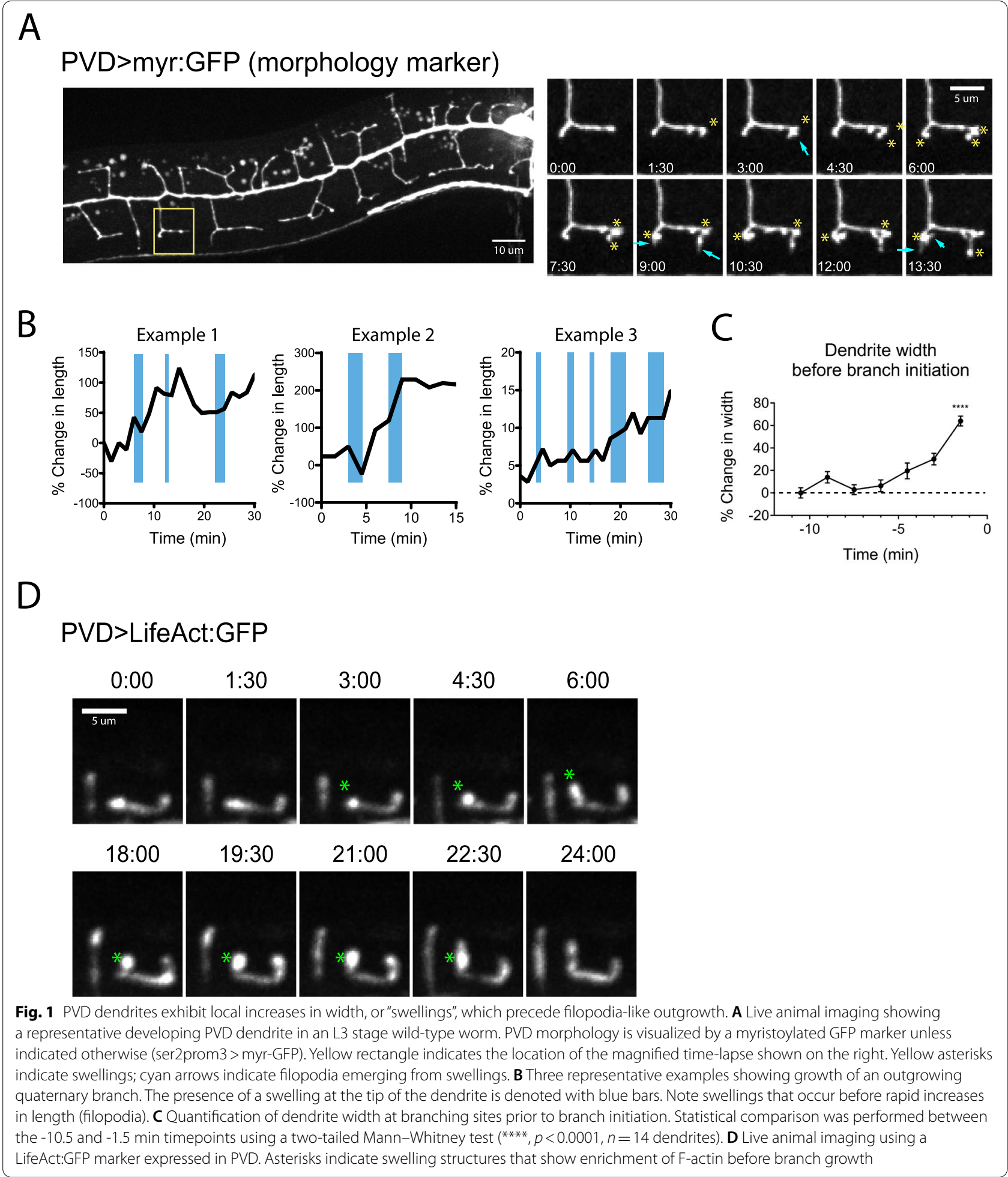

is enriched in these swellings prior to initiation or elongation of the dendritic branches (Fig. 1D). These data suggest that the swellings are actin-based, which is consistent with previous reports that PVD dendrite growth is primarily driven by actin polymerization [23, 24]. Finally, we noticed that filopodia grew from the swellings in various directions, but only a subset of the filopodia were stabilized as quaternary dendrites (see Additional files 1 and 
4). This behavior is consistent with the notion that filopodia are used to probe extracellular environments, perhaps in search of external guidance cues such as the patterned SAX-7 stripes along which PVD grows [27].

\section{Filopodia formation during PVD dendrite outgrowth depends on unc-34 (Ena/VASP) and unc-115 (abLIM)}

To identify the molecular mechanisms underlying the formation of the actin-rich swellings and filopodia observed above, we next examined PVD outgrowth in mutants of various actin regulators. We first focused on unc-34, the sole $C$. elegans Ena/VASP protein homolog, and $u n c-115$, an actin-binding LIM protein (abLIM) previously implicated in neurite outgrowth. Ena/VASP proteins play a critical role in polymerizing linear $\mathrm{F}$-actin to initiate and extend filopodia [28]. UNC-115 is thought to be a Rac effector that can act downstream of Netrin [9, 29] and has been shown to regulate axon guidance by affecting the dynamics of filopodia and lamellipodia in axonal growth cones $[9,30,31]$. Specifically, overexpression of membrane-targeted UNC-115 caused the formation of ectopic neurites, lamellipodia, and filopodia in the C. elegans PDE neuron, and deletion of the actinbinding VHD domain greatly reduced these effects [30]. We found that null mutants of unc-34 and null mutants of unc-115 both exhibited a strong loss of quaternary branches (Fig. 2A-B), suggesting that these actin regulators are also required for reorganizing the actin cytoskeleton during PVD dendrite morphogenesis.

We next used live animal imaging to examine if the unc-34 or unc-115 mutants affected swelling or filopodia formation during PVD dendrite outgrowth (Fig. 2CI; Additional files 2 and 3). We found that in both unc-34 and unc-115 mutants, filopodia formation was drastically reduced, whereas dendrite swellings remained unaffected (Fig. 2E-F). Furthermore, lack of filopodia in unc-34 or unc-115 mutants switched the wild-type "rapid-growth-with-pauses" mode of dendrite extension to a slow and steady outgrowth mode characterized by reduced speed of remaining outgrowth events (Fig. 2D, I, Fig. S1), and less variability in the speed of such outgrowth (Fig. $2 \mathrm{H}$ ). In particular, unc-34 mutants exhibited large, persistent growth cones at the tip of growing dendrites, which occasionally split to create new dendritic branches (Fig. 2C, Fig. S1, Additional file 2). These growth cones were wider than wild type growth cones (Fig. 2G) and extended steadily forward (Fig. 2H), reminiscent of the increased persistence of lamellipodia observed in migrating fibroblasts depleted of Ena/VASP proteins [32]. Taken together, our results suggest that both UNC-34 and UNC-115 play a key role in filopodia formation during PVD dendrite branching. The fast-growing filopodia ensure efficient growth of dendritic arbors, especially in the initiation of new quaternary branches. Because $u n c-34$ and $u n c-115$ mutants have also been reported to disrupt filopodia initiation in axonal growth cones [33], similar cytoskeletal mechanisms may also be involved in axon development.

\section{Swelling formation during PVD dendrite outgrowth depends on the WAVE Regulatory Complex}

Because neither unc-34 nor unc-115 mutants reduced swellings, we next asked what molecular mechanisms are required to form these structures. We previously showed that the WAVE Regulatory Complex (WRC) plays a critical role in PVD dendrite development through its direct interaction with the guidance receptor complex that mediates PVD outgrowth [23]. Consistent with our previous results, we found that mutants in the WRC components Sra1, Nap1/Hem-2, or WAVE/Scar (named gex-2, gex-3, and wve-1 in C. elegans, respectively) all showed severely truncated dendritic arbors at the L4 stage (Fig. 3A-B). All three WRC mutants exhibited a maternal effect lethal phenotype, where the offspring of heterozygous mothers are viable but sterile, suggesting that the alleles are indeed strong loss-of-function [34]. However, because the maternal WRC is clearly sufficient for the animals to develop to adulthood, it is possible that variation in the amount of residual WRC activity may account for slight differences in phenotype across the three mutants, such as the slightly weaker phenotype of the wve-1 mutant (Fig. 3B).

To test if the WRC is required to form swellings, we performed live animal imaging on gex-3 mutants and found that tertiary dendrites failed to form both swellings and filopodia (Fig. 3E-F; Additional file 5). As a result, we observed very few growth events overall (Fig. 3G-H; compare Additional files 4 and 5). We note that the gex3 phenotype is distinct from the phenotypes of unc-34 and unc-115 mutants in that both swellings and filopodia were absent in gex-3 mutants, whereas only filopodia, but not swellings, were disrupted in $u n c-34$ and $u n c-115$ mutants. Since filopodia arise from swellings in wild-type animals, it is plausible that the WRC promotes the formation of swellings, which then determines the locations for filopodia growth driven by UNC-34 and UNC-115. A similar mechanism, termed the "convergent elongation" model, has previously been proposed to describe filopodia formation from branched actin networks [35]. In this model, actin filaments from a branched network that is bundled together to form nascent filopodia. This mechanism has been proposed to explain filopodia formation in ex vivo chick sensory axons and cultured primary neuron growth cones [36, 37]. 


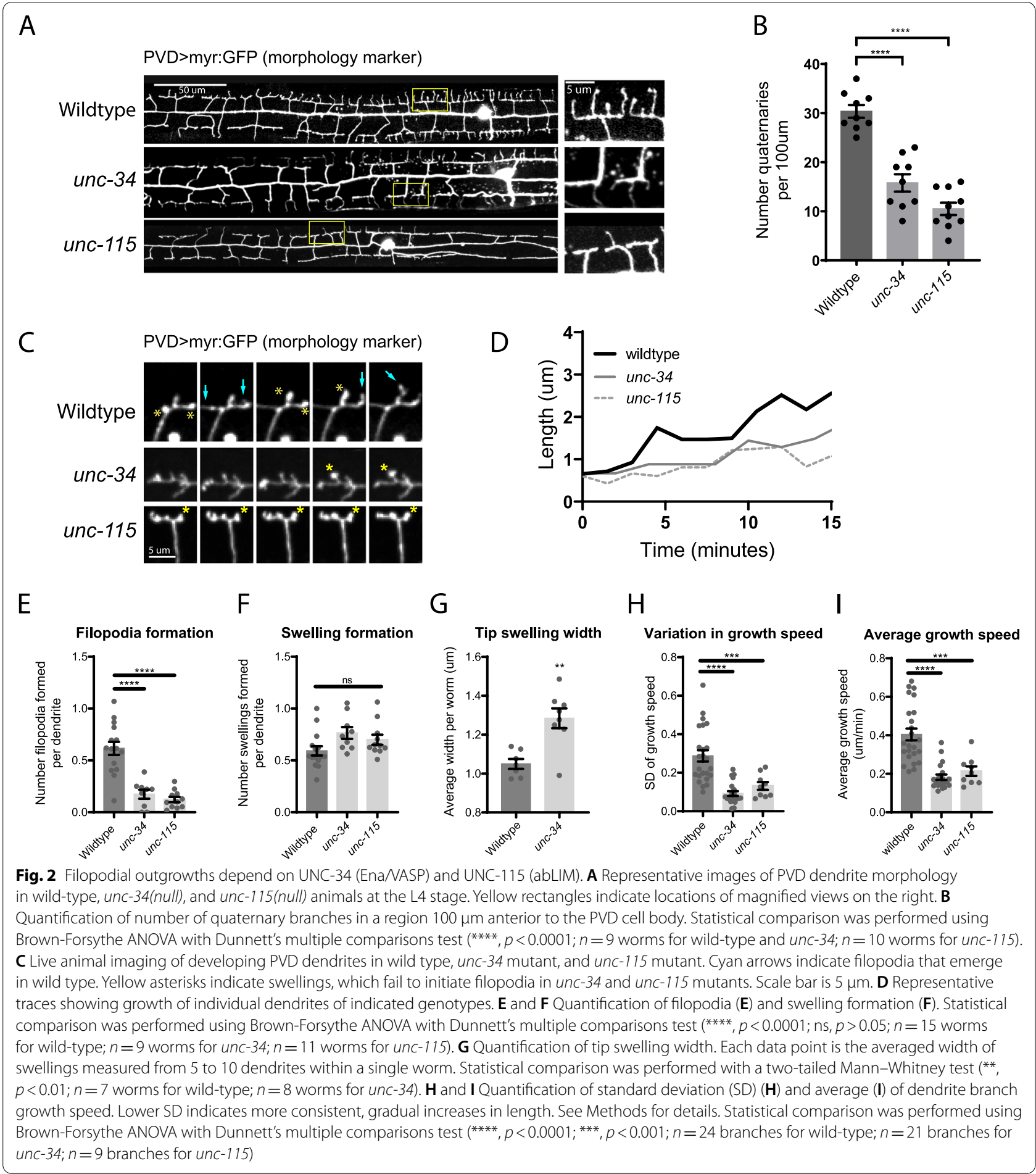

The WAVE Regulatory Complex can directly bind to UNC-34 through PPR-EVH1 interactions

What mechanisms might coordinate the local WRC activity in swellings with the UNC-34 or UNC-115 activity in filopodia formation? One possibility is that the
WRC recruits Ena/VASP proteins through a direct interaction between the poly-proline region (PPR) of Abi and WVE, and the EVH1 domain of Ena/VASP. Such an interaction was previously observed between human and Drosophila proteins, but the exact PPR sequences in Abi 


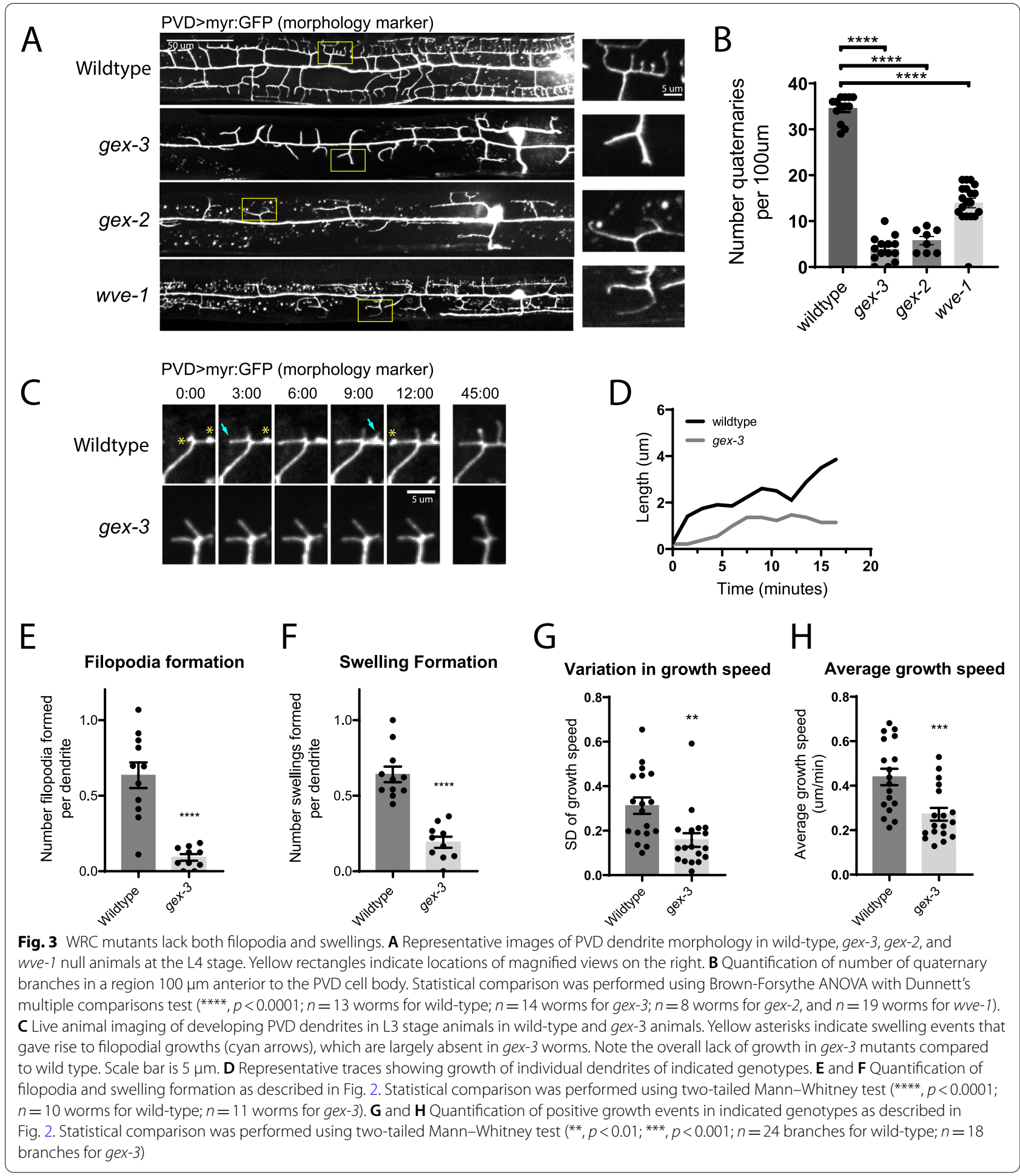

and WVE are divergent between various animal species [12].

To verify whether the UNC-34 EVH1 domain also directly binds to the $C$. elegans WRC (ceWRC), we recombinantly purified MBP (Maltose binding protein)tagged full-length (FL) Abi and WVE, MBP-tagged fragments of Abi and WVE, and a GST-tagged EVH1 domain of UNC-34 for pull-down assays (Fig. 4A). Consistent 


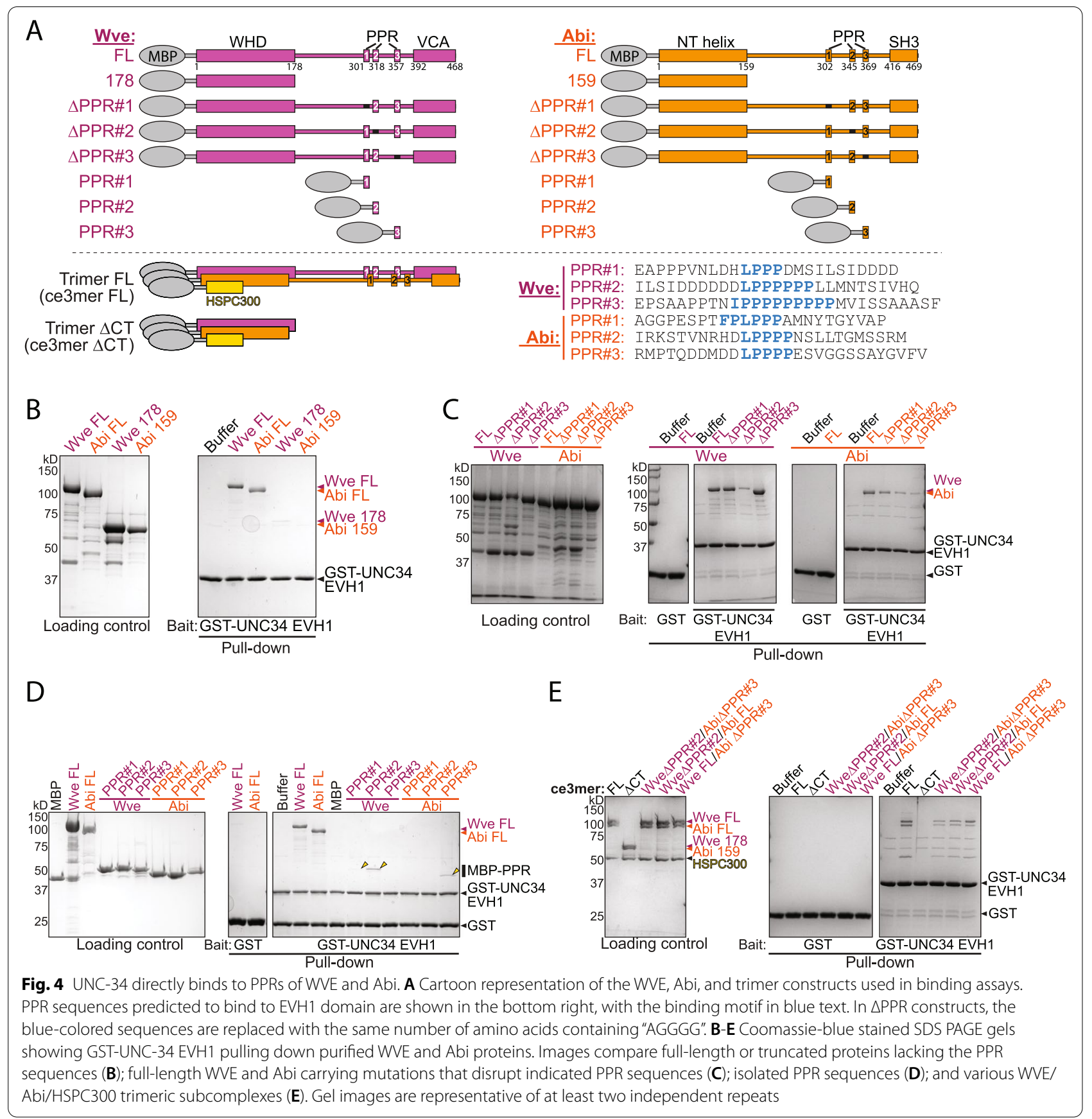

with previous reports [12, 28], GST-UNC34 EVH1 retained both Abi FL and WVE FL (Fig. 4B), but not truncated proteins that lack the PPR sequences (WVE 178 and Abi 159, Fig. 4B). By mutating each of the three PPR sequences in WVE and $\mathrm{Abi}$, which we predicted to bind to EVH1 using the "LPPPP" motif [12], we determined that multiple PPR sequences in both WVE and Abi contributed to the binding to EVH1, albeit at different levels. Particularly, deletion of WVE PPR\#2, Abi
PPR\#3, and to a lesser extent Abi PPR\#1 or PPR\#2, clearly decreased the binding of UNC-34 EVH1 (WVE $\triangle \mathrm{PPR} \# 2$, Abi $\triangle P P R \# 1,2$ \& 3; Fig. 4A, C). Note that although deleting WVE PPR\# 1 or \#3 did not appear to reduce the binding, deletion of WVE PPR\#2 still showed a residual, but clear interaction, suggesting PPR\#1 and \#3 remaining in the full-length WVE had a weak, but specific contribution to the binding. This result was further confirmed by direct binding between GST-UNC-34 EVH1 and isolated 
fragments containing individual WVE PPR\#2 or Abi PPR\#3 (Fig. 4A, D). In addition, we observed weak, but specific interaction of the isolated WVE PPR\#1 (Fig. 4D). We could not detect binding of the isolated WVE PPR\#3, Abi PPR\#1 or \#2, likely due to the limit of pull-down assays in detecting weak interactions of affinity in, empirically, tens of micromolar range (Fig. 4D). The slight discrepancy between full-length proteins, which contain multiple PPRs, and isolated PPRs likely reflects an avidity effect of multivalent interactions in sustaining a binding through otherwise weak individual ones.

Finally, we tested if UNC-34 EVH1 bound to the trimeric subcomplex of the WRC containing Abi, WVE, and HSPC300 (ce3mer; Fig. 4A). We used the trimeric subcomplex as a surrogate for the whole ceWRC pentamer because the subcomplex contained all PPRs found in the ceWRC pentamer, behaved well in biochemical reconstitution, and was less difficult to purify than the pentameric complex [23]. We found that in the context of the ceWRC subcomplex, entirely removing the PPR regions from both WVE and Abi abolished the binding to UNC34 EVH1, whereas mutating either WVE PPR\#2, or Abi PPR\#3, or both, partially reduced binding (Fig. 4E). This result further supports the notion that multiple WVE and Abi PPR sequences interact with UNC-34 EVH1. Although individual PPRs have different, sometimes weak, affinity (Fig. 4B-C), the availability of many PPRs in the trimer provides avidity (and possibly some cooperativity) to sustain a robust binding to EVH1, which could overcome deletion of the two PPRs with strongest affinity (Fig. 4E). Taken together, we conclude that the ceWRC can directly bind to UNC-34 through multiple PPR-EVH1 interactions.

\section{The EVH1 domain of UNC-34 is necessary for UNC-34 localization and function in vivo}

Having established that the ceWRC can bind to UNC34 in vitro, we next asked whether the WRC-UNC-34 interaction is necessary for recruiting UNC-34 in PVD dendrites in vivo. To do so, we examined UNC-34 localization during PVD outgrowth by expressing an UNC34:GFP fusion protein with a PVD-specific promoter in unc-34 null animals (Fig. 5A). We found that, consistent with the importance of Ena/VASP proteins in elongating actin bundles, and with a recent report describing UNC34 localization in PVD [24], UNC-34:GFP was enriched as puncta at the tips of growing dendrites (Fig. 5B, F; Additional file 6). Remarkably, UNC-34:GFP became enriched at the swelling sites before new branches started to emerge (Fig. 5D-F; Additional file 6). In contrast, when we expressed an UNC-34 lacking the EVH1 domain (UNC-34 $\triangle$ EVH:GFP, Fig. 5A), the localization at both the tips of growing dendrites and the swelling sites before new branch initiation was significantly reduced (Fig. 5C-F; Additional file 7). Both wildtype UNC-34:GFP and UNC-34 $\triangle E V H: G F P$ exhibit a decrease in signal after branch initiation (Fig. 5D) as a result of photobleaching during the imaging session. Note that removing the EVH1 domain from UNC-34 did not completely diminish its enrichment at the dendrite tips or swelling sites, which could be due other sequences in UNC-34, such as the EVH2 domain, binding the enriched F-actin [38, 39]. This interaction could account for the slight increase in UNC-34 $\triangle$ EVH:GFP signal at $1 \mathrm{~min}$ before branch initiation (Fig. 5D). Nevertheless, the reduced enrichment of UNC-34 $\triangle E V H: G F P$ at the swelling sites suggests the WRC-UNC-34 interaction plays an important role in recruiting UNC-34 to initiate filopodia formation. Finally, using the same constructs expressed in unc-34 null animals with a PVD morphology marker, we asked whether the WRC-UNC-34 interaction was required for the ability of UNC-34 to promote PVD dendrite growth. We found that while the wild-type UNC-34:GFP construct significantly rescued quaternary branch formation, UNC-34 $\triangle$ EVH:GFP showed no rescuing activity (Fig. 6A-B). Using live animal imaging, we found that the wild-type UNC-34:GFP rescue increased the occurrence of filopodia per dendrite, while UNC-34 $\triangle \mathrm{EVH}$ :GFP

(See figure on next page.)

Fig. 5 UNC-34 EVH1 domain is required for UNC-34 localization. A Schematic showing UNC-34 constructs used in the rescue experiments. B and C Live animal imaging of developing PVD dendrites in L3 stage wild-type animals that express wild-type UNC-34:GFP (B), or unc-34 null animals that express UNC-34 $\triangle \mathrm{EVH}: G F P(\mathbf{C})$. PVD dendrite morphology was visualized using a myristoylated-mCherry morphology marker (ser2prom3 > myr-mCh). White arrows indicate tip localization of UNC-34:GFP in filopodia. White arrowheads indicate swelling localization of UNC-34:GFP before filopodia initiation. White asterisk indicates large growth cone-mediated growth lacking UNC-34 $\triangle$ EVH:GFP enrichment at tip. Scale bar is $2 \mu \mathrm{m}$. Kymographs of dendrite growth are presented on the right. $\mathbf{D}$ and $\mathbf{E}$ Quantification of UNC-34:GFP enrichment to swellings before branch initiation. GFP intensity was measured at branching sites prior to branch outgrowth. Note the peak of UNC-34:GFP signal at 1 min before branch formation is absent in worms expressing the $\triangle E V H$ construct. Statistical comparison was performed between the -1.75 min (baseline) and -1.0 min timepoints using the Wilcoxon matched-pairs signed rank test. ( ${ }^{* *}, p<0.001 ; n s, p>0.05 ; n=28$ branches for wild-type UNC-34:GFP; $n=25$ branches for UNC-34 $\triangle$ EVH:GFP). F Quantification of UNC-34:GFP enrichment at dendrite tips. GFP intensity was measured at the tip of a growing dendrite and normalized as \% increase in intensity compared to GFP intensity along the dendrite shaft. Each data point is the average measured from 4-10 branches from a single worm. Statistical comparison was performed using the two-tailed Mann-Whitney test ${ }^{*}, p<0.05 ; n=8$ worms for wild-type, $n=9$ worms for UNC-34 $\triangle$ EVH:GFP) 
A

\begin{tabular}{|c|c|c|}
\hline UNC-34:GFP & \begin{tabular}{|lll} 
EVH1 & PPR & EVH2 \\
\end{tabular} & GFP \\
\hline & $198 \quad 256267 \quad 468$ & \\
\hline UNC-34 $\Delta$ EVH:GFF & $\mathrm{EVH} 2$ & GFP \\
\hline
\end{tabular}

B

PVD>UNC-34:GFP

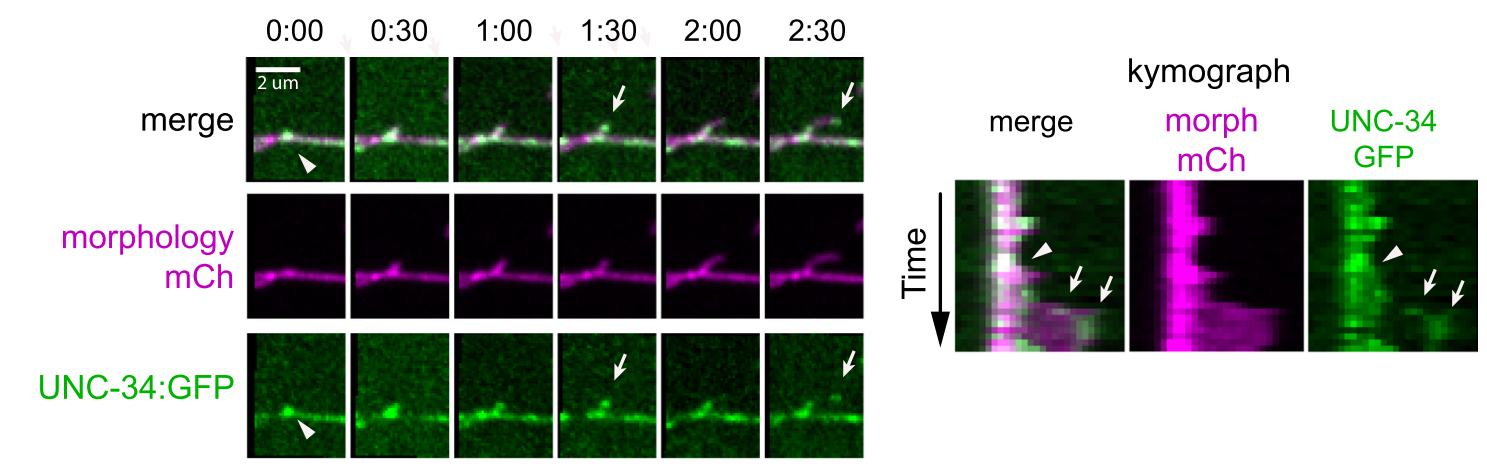

C

PVD>UNC-34 $\triangle E V H: G F P$

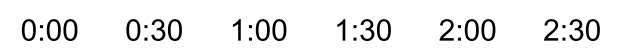

merge
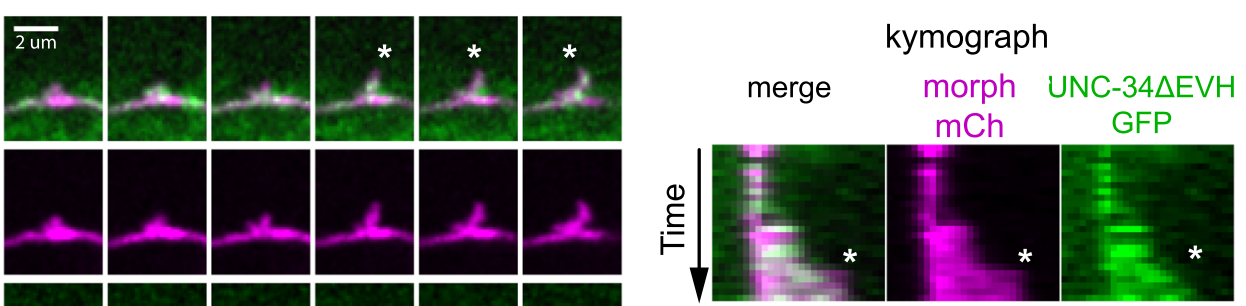

UNC-34AEVH:GFP
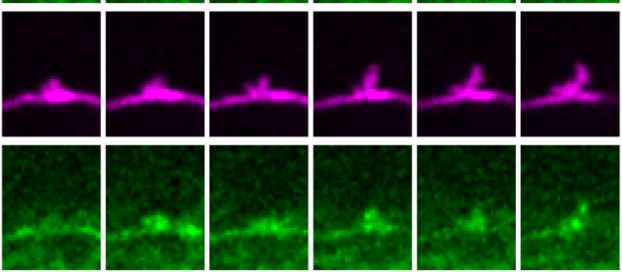

D
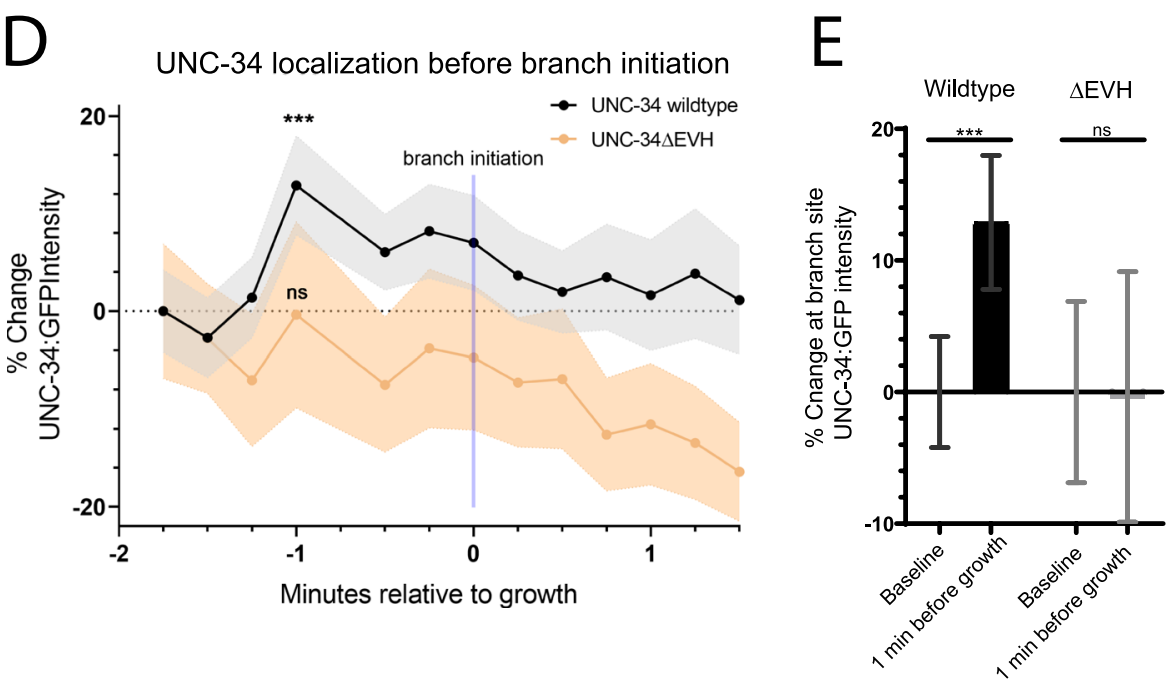

Fig. 5 (See legend on previous page.)

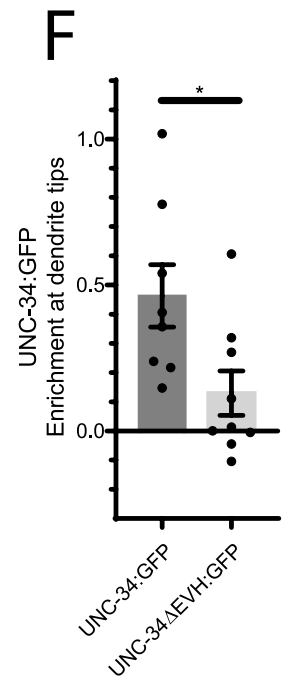




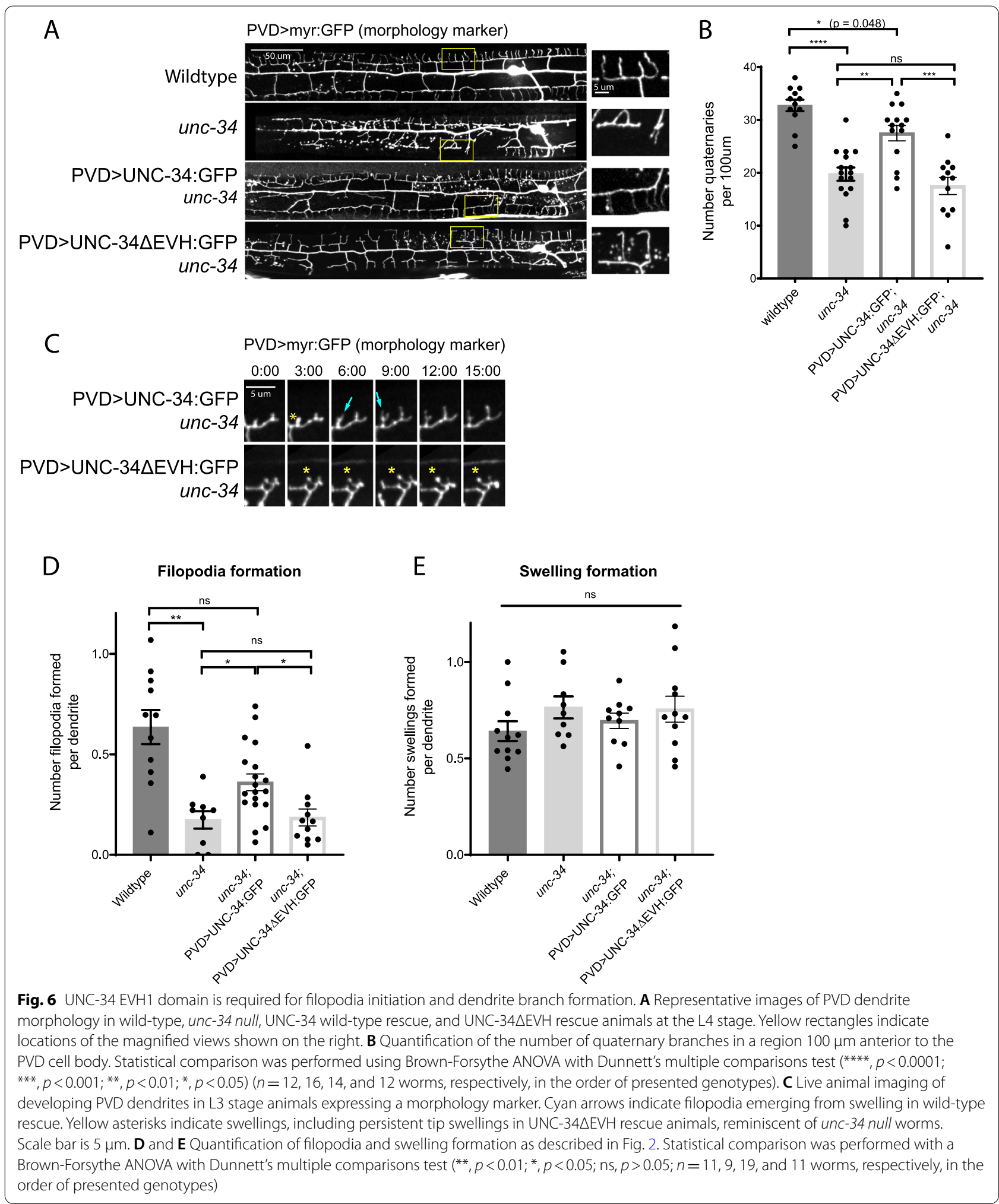




\section{Swellings form on existing branches due to WRC activity}

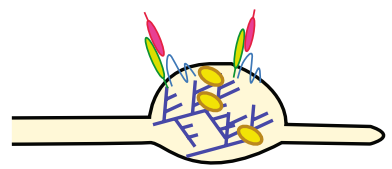

3. Guidance receptors promote new swelling formation through WRC recruitment

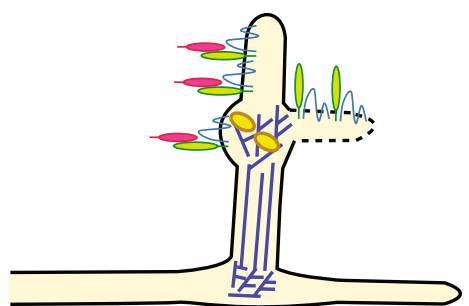

2. Filopodia grow and initiate new branches via UNC-34 and UNC-115 recruitment

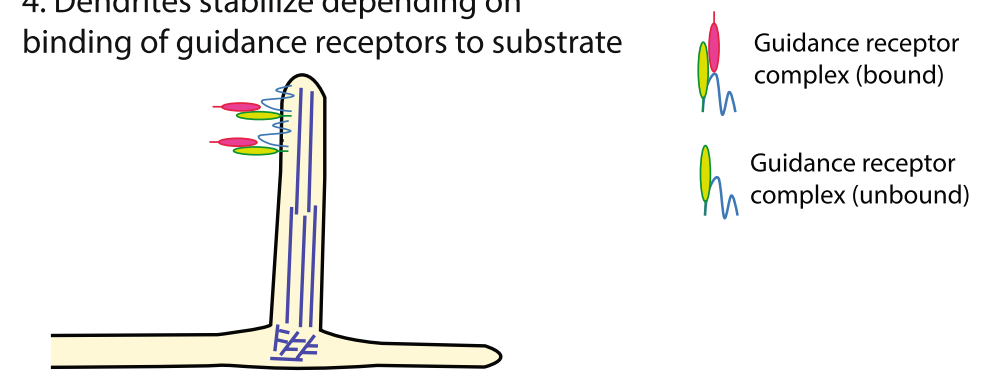

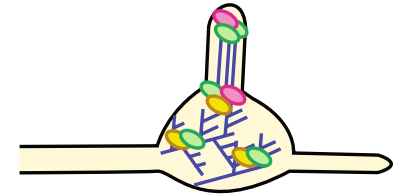

4. Dendrites stabilize depending on binding of guidance receptors to substrate
WRC

UNC-34 (Ena/VASP)

UNC-115 (abLIM)

||l F-actin

Guidance receptor complex (bound)

Guidance receptor h complex (unbound)

Fig. 7 A two-step model for PVD dendrite development. 1) Guidance receptor complexes first recruit and activate the WRC, which in turn produces a branched actin network resulting in the formation of swellings. 2) The WRC (likely together with the branched actin network) recruits UNC-34 to the swellings through PRR-EVH1 interactions, which then, together with UNC-115, produce linear actin bundles to initiate filopodia outgrowth. UNC-34 continues to elongate actin filaments at the tips of filopodia. 3) As the new branch elongates, guidance receptor complexes can continue to recruit the WRC to the growth tip, causing pauses, new swellings, and new filopodia. 4) Filopodia are finally stabilized as new dendrites when guidance receptors bind to extracellular ligands, or retracted when ligands are not present

failed to do so (Fig. 6C-D; Additional files 8 and 9). In comparison, formation of swellings remained unchanged in all above unc-34 null and rescued animals (Fig. 6E). Taken together, the above data support a model in which the WRC recruits UNC-34 to the swellings through the PPR-EVH1 interactions, and UNC-34 in turn promotes filopodia formation to initiate dendrite branching.

\section{Discussion}

Our data explain how two distinct actin regulators, the WRC and Ena/VASP, coordinate their actin polymerization activity to drive dendritic branch formation (Fig. 7). In our model, the PVD guidance receptor complex first recruits the WRC through the cytosolic domains of the receptors DMA-1 and HPO-30 [23]. Activation of the WRC creates a branched actin network and morphological swellings akin to lamellipodia of a growth cone. The WRC further recruits UNC-34 to the swellings through direct PPR-EVH1 interactions. The branched actin filaments at swellings can then be bundled and extended by UNC-115/abLIM and UNC-34/Ena/VASP, creating filopodial structures to initiate and extend dendritic branches. The rapid extension of filopodia is likely mediated by the potent actin polymerization activity of UNC-34/Ena/VASP [12]. Once quaternary branches begin extending, additional interactions between external cues and the PVD guidance receptors stabilize the newly formed dendrite and further recruit the WRC, creating an iterative process to build and establish a dendritic arbor.

The observed pauses during branch extension are likely due to the WRC being recruited and activated by the guidance receptors DMA-1 and HPO- 30 . In addition to physical recruitment by these guidance receptors, WRC activation likely depends on additional regulation within the PVD dendrite, such as local Rac activation. Notably, prior work has established that TIAM-1/Tiam1, a Rac GEF, is recruited by the PVD guidance receptor DMA-1 $[23,25]$. While the GEF activity of TIAM-1 does not seem to be necessary for its function, it is possible that TIAM-1 recruitment to the guidance receptor complex may help concentrate Rac near the WRC in order to promote WRC activation. Finally, while single mutants in other known WRC regulators such as Abl and Arf-6 [40, 41] have shown no obvious PVD phenotypes (data not shown), it remains possible that WRC activation can be regulated by other pathways that have yet to be identified during PVD dendrite morphogenesis. Following activation, the WRC likely subsequently recruits UNC-34/Ena/VASP to prime the actin networks for the next phase of dendrite extension. 


\section{Diverse roles for Ena/VASP in dendrite morphogenesis}

Recently, UNC-34 has also been reported to function in PVD dendrite morphogenesis by mediating self-avoidance between sister dendrites, functioning downstream of UNC-6/Netrin [24]. Here, our observations that unc34 mutants have fewer quaternary branches and greatly reduced filopodia formation during outgrowth suggest that UNC-34 acts cell-autonomously within PVD to promote dendrite outgrowth as well. We note that unc34 mutants often had primary dendrites that deviated from their normal trajectory to approach or merge with the sub-lateral line on which tertiary dendrites normally grow (Fig. 6A). This "wandering primary" phenotype was not consistently rescued by expressing UNC-34 in PVD, suggesting that UNC-34 might function in other cells, such as the ALA neuron [42, 43], to guide PVD primary dendrite development. Together, these observations indicate that Ena/VASP function is necessary for several aspects of neurite morphogenesis, including outgrowth, guidance, and self-avoidance.

\section{Swellings preconfigure dendrite branch sites}

The morphology and dynamics of our observed "swellings" resemble those of "actin blobs" described by Nithianandam and Chien [44], which were actin-rich structures found at dendrite branch emergence sites in Drosophila class IV da neurons. Like actin blobs, swellings in PVD were observed both along dendrite shafts to initiate nascent branches and at the tips of outgrowing dendrites to promote further growth. These similarities suggest that different types of neurons in animals may share a common general mechanism to initiate dendrite branching, although different systems may vary in exactly using what molecules to implement this mechanism (for example, WRC-Arp $2 / 3$ [17], cofilin [44], and Cobl [45] have all been found to be major players in creating new sites of branch formation). Based on this general mechanism, formation of enlarged dendrite loci that are rich in branched actin networks preconfigures dendrite branching sites. Branched actin filaments in the local actin-rich structures can then be converted into actin bundles to promote dynamic outgrowth of filopodia, which is reminiscent of the "convergent elongation model" describing filopodia formation [35] and our proposed model of filopodia protrusion from swelling sites in PVD.

\section{Conclusions}

In this study, we identified a series of cellular and molecular events that mediate the formation of a complex, branched dendritic arbor. We propose a mechanism by which the WRC establishes local actinrich dendritic swellings and directly recruits UNC-34/ Ena/VASP. Subsequently, UNC-34 builds filopodia and promotes rapid dendrite branching and outgrowth. Our findings are consistent with observations from Drosophila dendrite development, and suggest that the extension of linear, bundled actin from localized branched actin networks may be a general mechanism for initiating dendritic branches across different neuronal systems.

\section{Methods}

\section{C. elegans strains and maintenance}

C. elegans animals were raised on nematode growth medium (NGM) plates using OP50 Escherichia coli as a food source and maintained according to standard procedure unless otherwise noted [46]. Worms were raised at $20^{\circ} \mathrm{C}$ and N2 Bristol worms were used as the wildtype strain. Data for quantification of mature dendrite morphology (e.g., number of quaternary branches) were taken at the L4 stage. For dynamic imaging of developing dendrites, synchronized worms were grown from bleached eggs until L3 stage.

\section{DNA manipulations and transgenes}

Expression vectors were generated in the pSMdelta vector backbone (a derivative of pPD49.26).Transgenic $C$. elegans were prepared by microinjection into the gonad [47] using Pmyo $>m$ Cherry $(2 \mathrm{ng} / \mu \mathrm{l})$ as a co-injection marker. C. elegans strains used in this study are listed in Table 1.

\section{Confocal imaging of $\mathrm{C}$. elegans}

All images were acquired at room temperature in live C. elegans. For images of mature dendrite morphology, L4 stage animals were anesthesized using $10 \mathrm{mM} \mathrm{lev-}$ amisole in M9 buffer and mounted on 3\% agarose pads. Worms were then imaged on a 3i spinning disk microscope with a CSU-W1 spinning disk (Yokogawa) using a C-Apochromat 40x / 0.9 NA water immersion objective. Images were acquired as z-stacks $(0.75 \mu \mathrm{m} / \mathrm{step}, 15-18$ steps) to cover the entire PVD dendritic arbor, and maximum-intensity projections were used for subsequent analyses.

For dynamic imaging of developing dendrite morphology, L3 worms were mounted onto a glass-bottom imaging dish (MatTek) as follows: Worms were picked using an eyebrow pick into a droplet of $5 \mathrm{mM}$ levamisole on the glass bottom, and then covered with a $3 \%$ agarose pad. Worms were then imaged on a spinning disk microscope with a CSU-X1 spinning disk (Yokogawa) using a $40 \times$ objective. Images were acquired every $90 \mathrm{~s}$ as $\mathrm{z}$-stacks $(0.75 \mu \mathrm{m} / \mathrm{step}, 12$ steps $)$ to cover the entire PVD 
Table 1 Worm strains used in the study

\begin{tabular}{|c|c|}
\hline Strain Name & Genotype and description \\
\hline TV15911 & wyls592 [ser2prom3 $>$ myr-gfp + Podr-1 >rfp] \\
\hline TV24718 & unc-34(gm104); wyls592 \\
\hline TV17281 & unc-115(ky275); wyls592 \\
\hline TV17232 & gex-3(zu196) /nT1; wyls592 \\
\hline TV23509 & gex-2(ok1603)/dpy-9(e12); wyls592 \\
\hline TV17113 & wve-1(ok3308)/hT2; wdls51 [F49H12.4 promoter > gfp + unc-119(+)] \\
\hline TV24999 & $\begin{array}{l}\text { wyEx9925 [ser2prom3 > unc-34:gfp + Pmyo2 > mCherry]; wyls581 [ser2prom3 > myr- } \\
\text { mCherry + Podr-1 > gfp] }\end{array}$ \\
\hline TV26671 & wyEx10113 (ser2prom3 > UNC-34DEVH:GFP + Pmyo2 > mCherry); unc-34(gm 104); wyls581 \\
\hline TV24987 & wyEx9925; unc-34(gm 104); wyls592 \\
\hline TV25516 & wyEx10113 [ser2prom3 > unc-34AEVH:gfp + Pmyo2 > mCherry]; unc-34 (gm 104); wyls592 \\
\hline
\end{tabular}

dendritic arbor, and maximum-intensity projections were used for subsequent analyses.

For dynamic imaging of UNC-34:GFP, L3 worms were mounted onto a glass-bottom imaging dish as described above. Worms were imaged on a spinning disk microscope with a CSU-X1 spinning disk (Yokogawa) using a $63 \mathrm{x} / 1.4 \mathrm{NA}$ objective. Images were acquired every $15 \mathrm{~s}$ as z-stacks $(0.75 \mu \mathrm{m} / \mathrm{step}, 3$ steps $)$ to image a developing menorah, and maximum-intensity projections were used for subsequent analyses in Fiji.

\section{Quantification of dynamic imaging of dendrite morphology}

Quantification of morphological features was done as follows: Filopodia formation was measured by manually counting the number of filopodia formed within a 12-min timeframe and normalized to the number of existing dendrites at the beginning of the timeframe. Swelling formation was measured by manually counting the number of swellings present at the end of the same 12-min timeframe and normalized to the number of existing dendrites at the end of the frame.

Filopodia were visually identified as protrusions no wider than $0.54 \mu \mathrm{m}$ that extended at least $0.8 \mu \mathrm{m}$ between 1.5-min frames. Swellings along dendrite shafts were visually identified as local increases in branch width of at least $30 \%$, and occupying $0.54-1.4 \mu \mathrm{m}$ of the shaft. Swellings at dendrite tips were allowed to be larger than 1.4 $\mu \mathrm{m}$ to accommodate the expanded tip swellings in unc-34 mutants. Swellings were usually accompanied by a local increase in morphology marker intensity.

Quantification of growth speed was done as follows: Length of individual dendrites was tracked for at least $13 \mathrm{~min}$ at 1.5 -min time intervals. Positive increases in length between frames were identified as positive growth events for quantification. The standard deviation (SD) of such positive growth events was measured for each dendrite, and is presented in Figs. 2 and 3 as "Variation in growth speed". Lower SD indicates more consistent, gradual increases in dendrite length associated with lack of filopodia. The average of such positive growth events was also measured for each dendrite and is presented as "Average growth speed".

\section{Quantification of UNC-34:GFP localization}

UNC-34:GFP enrichment to swellings before branch initiation was measured as follows: GFP intensity was measured every $15 \mathrm{~s}$ at the location of a future branch initiation event starting 1.75 min before growth, and normalized to the average starting intensity across all measured dendrites. Statistical comparison was done between the $-1.75 \mathrm{~min}$ (baseline) and $-1.0 \mathrm{~min}$ timepoints.

UNC-34:GFP enrichment to dendrite tips was measured as \% increase in GFP intensity at the tip of a growing dendrite as compared to GFP intensity along the shaft of the dendrite. Values from 4-10 branches were averaged per worm, and average values from a single worm were used for statistical analysis.

\section{Statistical analysis}

All data are displayed as the mean \pm standard error of the mean (SEM). Statistical comparisons were conducted using two-tailed Mann-Whitney tests (to test for differences between two groups) or Brown-Forsythe ANOVA with Dunnett's multiple comparisons test (to test for differences between three or more groups). Sample sizes are indicated for each figure. ${ }^{* * * *}, p<0.0001$; ${ }^{* * * *}, p<0.001$; **, $p<0.01 ; *, p<0.05$ in all graphs. All statistical analyses and graph construction were performed using Prism 8.0 software (GraphPad Software, Inc).

\section{Protein purification and GST pulldown}

GST-UNC-34 EVH1, MBP-WVE, MBP-Abi, and MBPHSPC300 were expressed in Arctic Express (DE3) RIL 
(Agilent) or BL21 (DE3) $\mathrm{T} 1^{\mathrm{R}}$ (Sigma) cells after induction with $0.5 \mathrm{mM}$ IPTG at $10{ }^{\circ} \mathrm{C}$ or $18{ }^{\circ} \mathrm{C}$ for $16 \mathrm{~h}$. GST-UNC-34 EVH1 was purified through Glutathione Sepharose beads (GE Healthcare), followed by anion exchange chromatography using a Source $15 \mathrm{Q}$ column (GE Healthcare) at pH 7.0. MBP-WVE and MBP-Abi proteins were purified through amylose beads (New England Biolab). Trimeric subcomplexes containing WVE, $\mathrm{Abi}$, and HSPC300 were assembled and purified by following previously described protocols [12, 23]. Briefly, individually purified subunits were mixed at equal molar ratio in the presence of $1 \%(\mathrm{w} / \mathrm{v}) \mathrm{NP} 40$ and incubated on ice for $48 \mathrm{~h}$. The assembled trimer was then purified by anion exchange chromatography through a Source $15 \mathrm{Q}$ column at $\mathrm{pH}$ 8.0, cation exchange chromatography by a Source $15 \mathrm{~S}$ column at $\mathrm{pH} 6.0$, and size exclusion chromatography through a Superdex 200 column (GE Healthcare).

GST pull-down assays were performed as previously described [23]. Briefly, $20 \mu \mathrm{L}$ of GSH-Sepharose beads were mixed with 200 pmol of bait protein, 200 to 2,000 pmol of prey protein in $1 \mathrm{~mL}$ of pull-down buffer (50 or $100 \mathrm{mM} \mathrm{NaCl}, 10 \mathrm{mM}$ HEPES $\mathrm{pH} 7.0,5 \%$ (w/v) glycerol, $5 \mathrm{mM}$ 2-mercaptoethanol, and $0.05 \%$ Triton $\mathrm{X}-100)$. The samples were mixed at $4{ }^{\circ} \mathrm{C}$ for $30 \mathrm{~min}$, washed three times with each time using $1 \mathrm{~mL}$ of pulldown buffer, and eluted with $40 \mu \mathrm{L}$ of elution buffer containing $30 \mathrm{mM}$ reduced glutathione and $100 \mathrm{mM}$ Tris $\mathrm{pH}$ 8.5. The eluant was examined by SDS-PAGE and Coomassie blue staining.

\begin{abstract}
Abbreviations
Abi: Abelson interactor; abLIM: Actin-binding LIM protein; Ce3mer: A reconstituted trimer consisting of purified Abi, Wve, and HSPC300; Cobl: Cordon-bleu; da: Dendritic arborization; DCC: Deleted in Colorectal Carcinoma; DSCAM: Down syndrome cell adhesion molecule; Ena/NASP: Enabled/vasodilatorstimulated phosphoprotein; EVH: EnaNASP homology; FL: Full-length; GEF: Guanine nucleotide exchange factor; GFP: Green fluorescent protein; GST: Glutathione-S-transferase; HSPC300: Haematopoietic stem/progenitor cell protein 300; PPR: Polyproline region; WAVE (WVE): WASP-family verprolinhomologous protein; WRC: WAVE regulatory complex.
\end{abstract}

\section{Supplementary Information}

The online version contains supplementary material available at https://doi. org/10.1186/s13064-021-00154-0.

Additional file 1. Example branch time-lapse imaging of wild-type dendrite growth using a myr-GFP morphology marker. Images are taken every 90 seconds. Arrowhead tracks tip of an individual dendrite during outgrowth; asterisks indicate "swellings" along the dendrite. Scale bar is 5 $\mu \mathrm{m}$.

Additional file 2. Example branch time-lapse imaging of unc-34(null) dendrite growth using a myr-GFP morphology marker. Images are taken every 90 seconds. Yellow asterisks indicate persistent swellings that fail to generate filopodia and resemble lamellipodia-like growth cones. Scale bar is $5 \mu \mathrm{m}$.
Additional file 3. Example branch time-lapse imaging of unc-115(null) dendrite growth using a myr-GFP morphology marker. Images are taken every 90 seconds. Yellow asterisks indicate persistent swellings that fail to generate filopodia. Scale bar is $5 \mu \mathrm{m}$.

Additional file 4. Whole worm time-lapse imaging of wild-type dendrite growth using a myr-GFP morphology marker. Images are taken every 90 seconds. White arrowheads highlight three of many regions exhibiting quaternary dendrite growth. Magenta arrowhead highlights a secondary dendrite showing "rapid growth and pause" mode of growth, including swelling formation during pauses. Scale bar is $10 \mu \mathrm{m}$.

Additional file 5. Whole worm time-lapse imaging of gex-3(null) dendrite growth using a myr-GFP morphology marker. Images are taken every 90 seconds. Arrowheads point out dendrite growth events, although overall the dendritic arbor is much more static. Scale bar is $10 \mu \mathrm{m}$.

Additional file 6. Example branch time-lapse imaging of UNC-34:GFP localization using a myr-mCherry morphology marker. Images are taken every 15 seconds. White arrows indicate enrichment of UNC-34:GFP at tips of growing quaternary dendrites. White arrowheads indicate enrichment of UNC-34:GFP at branching sites prior to new branch initiation. Scale bar is $5 \mu \mathrm{m}$.

Additional file 7. Example branch time-lapse imaging of UNC$34 \triangle \mathrm{EVH}: G F P$ localization using a myr-mCherry morphology marker. Images are taken every 15 seconds. White asterisk highlights outgrowth of a quaternary dendrite with large, lamellipodia-like growth cone that lacks UNC-34 $\triangle E V H: G F P$ enrichment. Scale bar is $5 \mu \mathrm{m}$.

Additional file 8. Example branch time-lapse imaging of wild-type rescue experiment: UNC-34:GFP is expressed in an unc-34(null) worm. Dendrite growth is imaged using a myr-GFP morphology marker. Images are taken every 90 seconds. Yellow arrowheads indicate several filopodia events that emerge from swellings. Scale bar is $5 \mu \mathrm{m}$.

Additional file 9. Example branch time-lapse imaging of UNC-34 $\triangle E V H$ rescue experiment: UNC-34 $\triangle$ EVH:GFP is expressed in an unc-34(null) worm. Dendrite growth is imaged using a myr-GFP morphology marker. Images are taken every 90 seconds. Yellow asterisks indicate persistent swellings that fail to generate filopodia and resemble lamellipodia-like growth cones. Scale bar is $5 \mu \mathrm{m}$.

Additional file 10: Figure S1. Formation of swellings during dendrite outgrowth in mutants lacking filopodia. As in Fig. 1B, the presence of a swelling at the tip of the dendrite is denoted with blue bars. Representative examples are provided for wildtype (A), unc-34 mutant (B), and unc115 mutant (C) dendrites.

\section{Acknowledgements}

We thank all members of the Shen lab for helpful discussion, and Cen Gao, Karina Vega, and Victor (Blue) Paat for excellent technical support. We thank Drs. Gian Garriga, Cori Bargmann, James Priess, Robert Barstead, David Miller, and Liqun Luo for kindly sharing equipment and/or reagents.

\section{Authors' contributions}

R.S. contributed to study design, performed experiments and collected data, analyzed data, and wrote the primary manuscript. D.A.K. performed the protein purification and GST pull-down experiments, and contributed to the manuscript. B.C. contributed to study design and interpretation of data. K.S. contributed to study design and interpretation of data, and wrote the manuscript. All authors read, revised, and approved the final manuscript.

\section{Funding}

This work was supported by the Howard Hughes Medical Institute and the National Institute of Neurological Disorders and Stroke (1R01NS082208) to K.S.; the National Institute of General Medical Sciences (R35 GM128786) to B.C.; the National Science Foundation Graduate Research Fellowship and a training grant from the National Institutes of Health to R.S.

\section{Availability of data and materials}

All reagents and datasets used and/or analyzed during the current study are available from the corresponding author upon reasonable request. 


\section{Declarations}

Ethics approval and consent to participate

Not applicable.

\section{Consent for publication}

Not applicable.

\section{Competing interests}

The authors declare that they have no competing interests.

\section{Author details}

${ }^{1}$ Department of Biology, Stanford University, Stanford, CA 94305, USA. ${ }^{2} \mathrm{Neu}-$ rosciences IDP, Stanford University, Stanford, CA 94305, USA. ${ }^{3}$ Roy J. Carver Department of Biochemistry, Biophysics and Molecular Biology, lowa State University, Ames, IA 50011, USA. ${ }^{4}$ Howard Hughes Medical Institute, Stanford University, Stanford, CA 94305, USA

\section{Received: 9 May 2021 Accepted: 14 June 2021}

Published online: 19 July 2021

\section{References}

1. Jan Y-N, Jan LY. Branching out: mechanisms of dendritic arborization. Nat Rev Neurosci. 2010;11(5):316-28.

2. Dong $X$, Shen $K$, Bülow HE. Intrinsic and extrinsic mechanisms of dendritic morphogenesis. Annu Rev Physiol. 2015;1(77):271-300.

3. van Aelst L, Cline HT. Rho GTPases and activity-dependent dendrite development. Curr Opin Neurobiol. 2004;14:297-304. Elsevier Current Trends.

4. Ackerman SD, Perez-Catalan NA, Freeman MR, Doe CQ. Astrocytes close a motor circuit critical period. Nature. 2021;592(7854):414-20.

5. Chen Z, Borek D, Padrick SB, Gomez TS, Metlagel Z, Ismail AM, et al. Structure and control of the actin regulatory WAVE complex. Nature. 2010;468(7323):533-8.

6. Rotty JD, Wu C, Bear JE. New insights into the regulation and cellular functions of the ARP2/3 complex. Nat Rev Mol Cell Biol. 2013;14(1):7-12.

7. Lebrand C, Dent EW, Strasser GA, Lanier LM, Krause M, Svitkina TM, et al. Critical role of Ena/VASP proteins for filopodia formation in neurons and in function downstream of netrin-1. Neuron. 2004;42(1):37-49.

8. Krause M, Dent EW, Bear JE, Loureiro JJ, Gertler FB. Ena/VASP proteins: regulators of the actin cytoskeleton and cell migration. In: Annual review of cell and developmental biology. 2003. p. 541-64.

9. Gitai Z, Yu TW, Lundquist EA, Tessier-Lavigne M, Bargmann Cl. The netrin receptor UNC-40/DCC stimulates axon attraction and outgrowth through enabled and in parallel, Rac and UNC-115/abLIM. Neuron. 2003:37(1):53-65.

10. McConnell RE, van Veen JE, Vidaki M, Kwiatkowski AV, Meyer AS, Gertler FB. A requirement for filopodia extension toward Slit during Robo-mediated axon repulsion. J Cell Biol. 2016;213(2):261-74.

11. Bashaw GJ, Kidd T, Murray D, Pawson T, Goodman CS. Repulsive axon guidance: Abelson and enabled play opposing roles downstream of the roundabout receptor. Cell. 2000;101(7):703-15.

12. Chen XJ, Squarr AJ, Stephan R, Chen B, Higgins TE, Barry DJ, et al. Ena/ VASP proteins cooperate with the WAVE complex to regulate the actin cytoskeleton. Dev Cell. 2014;30(5):569-84.

13. Havrylenko S, Noguera P, Abou-Ghali M, Manzi J, Faqir F, Lamora A, et al. WAVE binds Ena/NASP for enhanced Arp2/3 complex-based actin assembly. Mol Biol Cell. 2015;26(1):55-65

14. Hayashi S, Inoue $Y$, Kiyonari H, Abe T, Misaki K, Moriguchi H, et al. Protocadherin-17 mediates collective axon extension by recruiting actin regulator complexes to interaxonal contacts. Dev Cell. 2014;30(6):673-87.

15. Korobova F, Svitkina TM. Molecular architecture of synaptic actin cytoskeleton in hippocampal neurons reveals a mechanism of dendritic spine morphogenesis. Mol Biol Cell. 2010;21(1):165-76.

16. Kannan R, Song JK, Karpova T, Clarke A, Shivalkar M, Wang B, et al. The Abl pathway bifurcates to balance Enabled and Rac signaling in axon patterning in Drosophila. Development (Cambridge). 2017;144(3):487-98.
17. Sturner T, Tatarnikova A, Mueller J, Schaffran B, Cuntz H, Zhang Y, et al. Transient localization of the Arp2/3 complex initiates neuronal dendrite branching in vivo. Development (Cambridge). 2019;146(7):dev171397.

18. Smith CJ, Watson JD, Spencer WC, O'Brien T, Cha B, Albeg A, et al. Timelapse imaging and cell-specific expression profiling reveal dynamic branching and molecular determinants of a multi-dendritic nociceptor in C. elegans. Dev Biol. 2010;345(1):18-33.

19. Dong X, Liu OW, Howell AS, Shen K. An extracellular adhesion molecule complex patterns dendritic branching and morphogenesis. Cell. 2013;155(2):296-307

20. Salzberg Y, Díaz-Balzac CA, Ramirez-Suarez NJ, Attreed M, Tecle E, Desbois $M$, et al. Skin-derived cues control arborization of sensory dendrites in Caenorhabditis elegans. Cell. 2013;155(2):308-20.

21. Zou W, Shen A, Dong X, Tugizova M, Xiang YK, Shen K. A multi-protein receptor-ligand complex underlies combinatorial dendrite guidance choices in C. elegans. Elife. 2016;5:e18345.

22. Díaz-Balzac CA, Rahman M, Lázaro-Peña MI, Martin Hernandez LA Salzberg Y, Aguirre-Chen C, et al. Muscle- and skin-derived cues jointly orchestrate patterning of somatosensory dendrites. Curr Biol. 2016;26(17):2379-87.

23. Zou W, Dong X, Broederdorf TR, Shen A, Kramer DA, Shi R, et al. A dendritic guidance receptor complex brings together distinct actin regulators to drive efficient F-actin assembly and branching. Dev Cell. 2018:45(3):362-375.e3.

24. Sundararajan L, Smith CJ, Watson JD, Millis BA, Tyska MJ, Miller DM. Actin assembly and non-muscle myosin activity drive dendrite retraction in an UNC-6/Netrin dependent self-avoidance response. Ye B, editor. PLoS Genet. 2019;15(6):e1008228.

25. Tang LT, Diaz-Balzac CA, Rahman M, Ramirez-Suarez NJ, Salzberg Y, Lázaro-Peña MI, et al. TIAM-1/GEF can shape somatosensory dendrites independently of its GEF activity by regulating F-actin localization. Elife. 2019:8:e38949.

26. Salzberg Y, Ramirez-Suarez NJ, Bülow HE. The proprotein convertase KPC-1/furin controls branching and self-avoidance of sensory dendrites in Caenorhabditis elegans. PLoS Genet. 2014;10(9):e1004657.

27. Liang X, Dong X, Moerman DG, Shen K, Wang X. Sarcomeres pattern proprioceptive sensory dendritic endings through UNC-52/Perlecan in C.elegans. Dev Cell. 2015;33(4):388-400.

28. Bear JE, Gertler FB. Ena/NASP: towards resolving a pointed controversy at the barbed end. J Cell Sci. 2009;122(12):1947-53.

29. Struckhoff EC, Lundquist EA. The actin-binding protein UNC-115 is an effector of Rac signalling during axon pathfinding in C. elegans. Development. 2003;130:693-704

30. Yang Y, Lundquist EA. The actin-binding protein UNC-115/abLIM controls formation of lamellipodia and filopodia and neuronal morphogenesis in Caenorhabditis elegans. Mol Cell Biol. 2005:25(12):5158-70.

31. Lundquist EA, Herman RK, Shaw JE, Bargmann CI. UNC-115, a conserved protein with predicted LIM and actin-binding domains, mediates axon guidance in C. elegans. Neuron. 1998;21(2):385-92

32. Bear JE, Svitkina TM, Krause M, Schafer DA, Loureiro JJ, Strasser GA, et al. Antagonism between Ena/VASP proteins and actin filament capping regulates fibroblast motility. Cell. 2002;109(4):509-21.

33. Norris AD, Dyer JO, Lundquist EA. The Arp2/3 complex, UNC-115/abLIM, and UNC-34/Enabled regulate axon guidance and growth cone filopodia formation in Caenorhabditis elegans. Neural Dev. 2009;4(1):38.

34. Soto MC, Qadota H, Kasuya K, Inoue M, Tsuboi D, Mello CC, et al. The GEX-2 and GEX-3 proteins are required for tissue morphogenesis and cell migrations in C. elegans. Genes Dev. 2002;16(5):620-32.

35. Svitkina TM, Bulanova EA, Chaga OY, Vignjevic DM, Kojima SI, Vasiliev JM, et al. Mechanism of filopodia initiation by reorganization of a dendritic network. J Cell Biol. 2003;160(3):409-21.

36. Korobova F, Svitkina T. Arp2/3 complex is important for filopodia formation, growth cone motility, and neuritogenesis in neuronal cells. Mol Biol Cell. 2008;19(4):1561-74.

37. Spillane M, Ketschek A, Jones SL, Korobova F, Marsick B, Lanier L, et al. The actin nucleating Arp2/3 complex contributes to the formation of axonal filopodia and branches through the regulation of actin patch precursors to filopodia. Dev Neurobiol. 2011;71(9):747-58. 
38. Bachmann C, Fischer $L$, Walter $U$, Reinhard M. The EVH2 domain of the vasodilator-stimulated phosphoprotein mediates tetramerization, F-actin binding, and actin bundle formation. J Biol Chem. 1999;274(33):23549-57

39. Nakagawa H, Miki H, Ito M, Ohashi K, Takenawa T, Miyamoto S. N-WASP, WAVE and Mena play different roles in the organization of actin cytoskeleton in lamellipodia. J Cell Sci. 2001;114(8):1555-65.

40. Singh V, Davidson AC, Hume PJ, Humphreys D, Koronakis V. Arf GTPase interplay with Rho GTPases in regulation of the actin cytoskeleton. Small GTPases. 2019;10(6):411-18.

41. Mendoza MC. Phosphoregulation of the WAVE regulatory complex and signal integration. Semin Cell Dev Biol. 2013;24(4):272-9.

42. Chen $\mathrm{CH}$, Hsu HW, Chang YH, Pan CL. Adhesive L1CAM-Robo signaling aligns growth cone F-actin dynamics to promote axon-dendrite fasciculation in C. elegans. Dev Cell. 2019;48(2):215-228.e5.

43. Ramirez-Suarez NJ, Belalcazar HM, Salazar CJ, Beyaz B, Raja B, Nguyen $K C Q$, et al. Axon-dependent patterning and maintenance of somatosensory dendritic arbors. Dev Cell. 2019;48(2):229-244.e4
44. Nithianandam V, Chien CT. Actin blobs prefigure dendrite branching sites. J Cell Biol. 2018:217(10):3731-46.

45. Hou W, Izadi M, Nemitz S, Haag N, Kessels MM, Qualmann B. The actin nucleator Cobl is controlled by calcium and calmodulin. Machesky L, editor. PLoS Biol. 2015;13(9):e1002233.

46. Brenner S. The genetics of Caenorhabditis elegans. Genetics. 1974;77(1):71-94

47. Mello C, Fire A. DNA transformation. Methods Cell Biol. 1995;48(C):451-82

\section{Publisher's Note}

Springer Nature remains neutral with regard to jurisdictional claims in published maps and institutional affiliations.
Ready to submit your research? Choose BMC and benefit from:

- fast, convenient online submission

- thorough peer review by experienced researchers in your field

- rapid publication on acceptance

- support for research data, including large and complex data types

- gold Open Access which fosters wider collaboration and increased citations

- maximum visibility for your research: over $100 \mathrm{M}$ website views per year

At BMC, research is always in progress.

Learn more biomedcentral.com/submissions 